\title{
GENERATORS FOR A MAXIMALLY DIFFERENTIAL IDEAL IN POSITIVE CHARACTERISTIC
}

\author{
ALOK KUMAR MALOO
}

\section{Introduction}

In this note we give the structure of maximally differential ideals in a Noetherian local ring of prime characteristic $p>0$, in terms of their generators. More precisely, we prove the following result:

THeorem 4. Let $A$ be a Noetherian local ring of prime characteristic $p>0$ with maximal ideal $m$. Let $I$ be a proper ideal of $A$. Suppose $n=\operatorname{emdim}(A)$ and $r=\operatorname{emdim}(A / I)$. If $I$ is maximally differential under a set of derivations of $A$ then there exists a minimal set $x_{1}, \ldots, x_{n}$ of generators of $m$ such that $I=\left(x_{1}^{p}, \ldots, x_{r}^{p}\right.$, $\left.x_{r+1}, \ldots, x_{n}\right)$.

This result was proved by the author in [3, Lemma 2.2], under the additional hypothesis that $A$ is complete and $I$ is maximally differential under a set of $k$-derivations of $A$, where $k$ is a coefficient field of $A$.

Using the methods we use to prove the above result we give a different proof for Harper's Theorem (as called by H. Matsumura, [Cf. [4, Theorem on p. 206]]). The following formulation of Harper's Theorem is due to S. Yuan [5]:

"Let $A$ be a differentially simple ring of positive characteristic $p$. Then $A$ is local. Let $\mathrm{m}$ be the maximal ideal of $A$ and let $n=\operatorname{dim}_{A / \mathrm{m}}\left(\mathrm{m} / \mathrm{m}^{2}\right)$. If $n<\infty$ then

$$
A \cong k\left[X_{1}, X_{2}, \ldots, X_{n}\right] /\left(X_{1}^{p}, X_{2}^{p}, \ldots, X_{n}^{p}\right),
$$

where $k$ is a field and $X_{1}, X_{2}, \ldots, X_{n}$ are indeterminates over $k$."

Our proof of Harper's Theorem is very straightforward and is much simpler than the original proof by L. Harper [1] and S. Yuan's proof, both of which involve somewhat complicated computations.

Received January 5, 1993. 


\section{The results}

By a ring we mean a commutative ring with 1 .

Let $A$ be a ring.

Let $\mathfrak{D}$ be a set of derivations of $A$. Then an ideal $I$ is called a $\mathfrak{D}$-differential ideal if $d(I) \subset I$ for all $d \in \mathfrak{D}$. An ideal $I$ is called a maximally $\mathfrak{D}$-differential ideal if it is a proper $\mathfrak{D}$-differential ideal and for every ideal $J$ of $A$ with $I \subsetneq J \subsetneq$ $A, J$ is not $\mathscr{D}$-differential. An ideal $I$ is called a maximally differential ideal if it is maximally $\mathfrak{D}$-differential for a set $\mathfrak{D}$ of derivations of $A$.

A ring is called a differentially simple ring if the ideal (0) is maximally differential in it.

For a derivation $d$ of $A$, by $d$-differential we mean $\{d\}$-differential.

. Lemma 1. Let $A$ be a ring of prime characteristic $p>0$. Let $\delta$ be a derivation of $A$ and let $x \in A$ such that $\delta(x)=1$. Then:

(a) Let $I$ be a $\delta$-differential ideal of $A$. If $a_{0}, a_{1}, \ldots, a_{p-1} \in \operatorname{ker}(\delta)$ such that $\sum_{i=0}^{p-1} a_{i} x^{i} \in I$ then $a_{i} \in I$ for all $i=0,1, \ldots, p-1$.

(b) Let $E=\sum_{i=0}^{p-1}(-x)^{i} \delta^{i} / i$ !. Then:

(i ) $\delta E=-x^{p-1} \delta^{p}$.

(ii) For erery $a \in A, E(x a)=-x^{p} \delta^{p-1}(a)$.

(iii) For every $a \in A, E^{2}(a) \equiv E(a)\left(\bmod \left(x^{p}\right)\right)$.

Proof. (a) Since $I$ is $\delta$-differential, $\delta^{p-1}\left(\sum_{i=0}^{p-1} a_{i} x^{i}\right)=(p-1) ! a_{p-1} \in I$. Hence $a_{p-1} \in I$. By induction, $a_{p-2}, \ldots, a_{0} \in I$.

(b) Statements (i) and (ii) are straightforward from the definition of $E$. Statement (iii) follows from (ii).

Proposition 2. Let $A, \delta, x$ and $E$ be as in Lemma 1. Suppose, in addition, $x^{p}=0$. Then $E$ is a ring homomorphism. Let $A_{0}=E(A)$. Then:

(i ) $A_{0}=\{a \in A \mid E(a)=a\}=\operatorname{ker}\left(\delta+x^{p-1} \delta^{p}\right)$.

(ii) $A^{p}=\left\{a^{p} \mid a \in A\right\} \subset A_{0}$ and $A$ is a free $A_{0}$-module with basis $1, x, \ldots$, $x^{p-1}$.

(iii) Let $\mathfrak{D}$ be a set of derivations of $A$ such that $\delta \in \mathscr{D}$ and let $I$ be a maximally D-differential ideal of $A$. Then $I_{0}=I \cap A_{0}$ is a maximally differential of $A_{0}$ and $I_{0} A=I$.

Proof. Since $x^{p}=0$, by Leibnitz rule, $E$ is a ring homomorphism. Put $\delta^{\prime}=\delta+x^{p-1} \delta^{p}$, then $\delta^{\prime}$ is a derivation of $A$. 
(i) Let $a \in A_{0}$. Then $a=E(b)$ for some $b \in A$. Therefore by Lemma 1 $E(a)=E E(b)=E(b)=a$, as $x^{p}=0$. Hence $A_{0}=\{a \in A \mid E(a)=a\}$. Now we prove the other equality. Let $a \in A$ such that $a=E(a)$. Then, by Lemma 1, $\delta(a)=\delta E(a)=-x^{p-1} \delta^{p}(a)$. Therefore $\delta^{\prime}(a)=0$. Conversely, let $a \in \operatorname{ker}\left(\delta^{\prime}\right)$. We show by induction that $x^{i} \delta^{i}(a)=0$ for $i=1, \ldots, p-1$. Since $x^{p}=0$, $x \delta(a)=0$. Suppose $x^{i} \delta^{i}(a)=0$ for $1 \leq i<p-1$. Then $0=\delta\left(x^{i+1} \delta^{i}(a)\right)=$ $x^{i+1} \delta^{i+1}(a)+(i+1) x^{i} \delta^{i}(a)=x^{i+1} \delta^{i+1}(a)$. Hence $E(a)=a$. Therefore $A_{0}=$ $\operatorname{ker}\left(\delta^{\prime}\right)$.

(ii) Since $\delta\left(a^{p}\right)=0$ for all $a \in A, A^{p} \subset A_{0}$. Now we show that $A$ is generated by $1, x, \ldots, x^{p-1}$ over $A_{0}$. Let $a_{0} \in A$. By induction on $i$ we construct $a_{i} \in A_{0}$ for $i=0,1, \ldots, p-1$ such that $a=\sum_{i=0}^{p-1} a_{i} x^{i}$. Now $a=E(a)+x b_{1}$ for some $b_{1} \in A$. Take $a_{0}=E(a)$ and $a_{1}=E\left(b_{1}\right)$. Again $b_{1}=E\left(b_{1}\right)+x b_{2}$ for some $b_{2} \in A$. Take $a_{2}=E\left(b_{2}\right)$ and so on. Since $x^{p}=0$ we have $a=\sum_{i=0}^{p-1} a_{i} x^{i}$. As $A_{0}=\operatorname{ker}\left(\delta^{\prime}\right)$ by (i) and $\delta^{\prime}(x)=1$, by Lemma $1,1, x, \ldots, x^{p-1}$ are linearly in. dependent over $A_{0}$.

(iii) Let $a \in I$. Then, by (ii), $a=\sum_{i=0}^{p-1} a_{i} x^{i}$, for some $a_{0}, a_{1}, \ldots, a_{p-1} \in A_{0}$. Since $I$ is $\delta^{\prime}$-differential, $\delta^{\prime}(x)=1$ and $A_{0}=\operatorname{ker}\left(\delta^{\prime}\right)$, by Lemma $1, a_{i} \in I$ for all $i=0,1, \ldots, p-1$. Hence $I=I_{0} A$.

Let $d \in \mathfrak{D}$. For $a \in A_{0}$, let $d_{i}(a)$ denote the coefficient of $x^{i}$ in the expression of $d(a), i=0,1, \ldots, p-1$. Then $d_{i}$ 's are derivations of $A_{0}$. (We have borrowed this construction from [2].) Let $\mathfrak{D}_{0}=\left\{d_{i} \mid d \in \mathfrak{D}, i=0,1, \ldots, p-1\right\}$. We show that $I_{0}$ is maximally $\mathfrak{D}_{0}$-differential. First we show that $I_{0}$ is $\mathfrak{D}_{0}$-differential. Let $a$ $\in I_{0}$ and $d \in \mathfrak{D}$. Since $a \in I, d(a)=\sum_{i=0}^{p-1} d_{i}(a) x^{i} \in I$. Therefore by Lemma 1 $d_{i}(a) \in I$. Hence $d_{i}(a) \in I_{0}$ for all $i=0,1, \ldots, p-1$ and $d \in \mathfrak{D}$. Therefore $I_{0}$ is $\mathfrak{D}_{0}$-differential. Let $J$ be a $\mathfrak{D}_{0}$-differential ideal of $A_{0}$ containing $I_{0}$. Let $a \in J$ and $d \in \mathfrak{D}$. Then $d(a)=\sum_{i=0}^{p-1} d_{i}(a) x^{i} \in J A$. Therefore $J A$ is $\mathfrak{D}$-differential. Since $I$ is maximally $\mathfrak{D}$-differential and $I=I_{0} A \subset J A$ either $J A=I$ or $J A=A$. Since $A$ is faithfully flat over $A_{0}, J=I_{0}$ or $J=A_{0}$ accordingly. Hence $I_{0}$ is a maximally differential ideal of $A_{0}$.

COROLlary 3 [Harper's Theorem, Cf. [1]]. Let $A$ be a differentially simple ring of positive characteristic $p$. Then $A$ is local. Let $\mathrm{m}$ be the maximal ideal of $A$ and let $n=\operatorname{dim}_{A / \mathrm{m}}\left(\mathrm{m} / \mathrm{m}^{2}\right)$. If $n<\infty$ then

$$
A \cong k\left[X_{1}, \ldots, X_{n}\right] /\left(X_{1}^{p}, \ldots, X_{n}^{p}\right),
$$

where $k$ is a field and $X_{1}, X_{2}, \ldots, X_{n}$ are indeterminates over $k$. 
Proof. Let $\mathfrak{D}$ be the set of all derivations of $A$. Then $(0)$ is maximally D-differential.

Let $K=\{a \mid d(a)=0$ for all $a \in \mathfrak{D}\}$. By differential simplicity of $A$ it follows that $K$ is a field. Hence $p$ is prime.

If $a \in A$ is a nonunit then $a^{p}=0$ as the ideal $\left(a^{p}\right)$ is $\mathfrak{D}$-differential. Hence $A$ is local of Krull dimension zero. We prove the result by induction on $n$. If $n=0$ then $\mathrm{m}=\mathrm{m}^{2}$. Hence $d(\mathrm{~m})=d\left(\mathrm{~m}^{2}\right) \subset \mathrm{m}$ for all $d \in \mathfrak{D}$. Therefore $\mathrm{m}$ is D-differential. Hence $m=(0)$, i.e., $A$ is a field. Suppose $n \geq 1$. Then there exist $d \in \mathfrak{D}$ and $x \in \mathfrak{m}$ such that $d(x) \notin \mathfrak{m}$. By replacing $d$ by $d(x)^{-1} d$, we may assume that $d(x)=1$. Since $x^{p}=0$, by Proposition 2 there exists a local subring $A_{0}$ of $A$ such that $A^{p} \subset A_{0}, A$ is a free $A_{0}$-module with basis $1, x, \ldots, x^{p-1}$ and (0) is maximally differential in $A_{0}$. Then $A \cong A_{0}[X] /\left(X^{p}\right)$ where $X$ is an indeterminate over $A_{0}$ and $A_{0} \cong A /(x)$. Let $\mathrm{m}_{0}$ be the maximal ideal of $A_{0}$. Now, $\operatorname{dim}_{A_{0} / \mathrm{m}_{0}}\left(\mathrm{~m}_{0} / \mathrm{m}_{0}^{2}\right)=\operatorname{dim}_{A / \mathrm{m}}\left(\mathrm{m} /(x)+\mathrm{m}^{2}\right)=n-1$ as $x \notin \mathrm{m}^{2}$. Therefore, by induction, we are through.

Theorem 4. Let $A$ be a Noetherian local ring of prime characteristic $p>0$ with maximal ideal $\mathrm{m}$. Let $I$ be a proper ideal of $A$. Suppose $n=\operatorname{emdim}(A)$ and $r=\operatorname{emdim}(A / I)$. If $I$ is maximally differential under a set of derivations of $A$ then there exists a minimal set $x_{1}, \ldots, x_{n}$ of generators of $m$ such that $I=\left(x_{1}^{p}, \ldots, x_{r}^{p}\right.$, $\left.x_{r+1}, \ldots, x_{n}\right)$.

Proof. Let $\mathfrak{D}$ denote the set of all derivations $d$ of $A$ such that $d(I) \subset I$. Then $I$ is maximally $\mathfrak{D}$-differential.

If $a \in \mathfrak{m}$ then $\left(a^{p}\right)$ is $\mathfrak{D}$-differential and hence $a^{p} \in I$.

We prove the result by induction on $r$. If $r=0$ then there is nothing to prove. Let $r \geq 1$. Then there exist $\delta \in \mathfrak{D}$ and $x \in \mathrm{m}$ such that $\delta(x) \notin \mathrm{m}$. By replacing $\delta$ by $(\delta(x))^{-1} \delta$ we may assume that $\delta(x)=1$. Let $B=A /\left(x^{p}\right), \mathfrak{n}=$ $\mathrm{m} /\left(x^{p}\right)$ and $J=I /\left(x^{p}\right)$. Let $y$ be the image of $x$ in $B$.

For $d \in \mathfrak{D}$, let $d^{\prime}$ denote the derivation on $B$ induced by $d$ and let $\mathfrak{D}^{\prime}=$ $\left\{d^{\prime} \mid d \in \mathfrak{D}\right\}$. Then $J$ is maximally $\mathfrak{D}^{\prime}$-differential in $B, \delta^{\prime}(y)=1$ and $y^{p}=0$. Therefore by Proposition 2 there exists a local subring $B_{0}$ of $B$ such that $B^{p} \subset$ $B_{0}, B$ is a free $B_{0}$-module with basis $1, y, \ldots, y^{p-1}, J_{0}=J \cap B_{0}$ is maximally differential in $B_{0}$ and $J=J_{0} B$. It is immediate from above data that $B_{0} \cong$ $B /(y) \cong A /(x)$ and $B_{0} / J_{0} \cong B / J+(y) \cong A / I+(x)$. Since $x \notin I+\mathrm{m}^{2}$ it follows that emdim $\left(B_{0}\right)=n-1$ and $\operatorname{emdim}\left(B_{0} / J_{0}\right)=r-1$. Hence by induction $J_{0}=\left(y_{1}^{p}, \ldots, y_{r-1}^{p}, y_{r}, \ldots, y_{n-1}\right)$ for a minimal set $y_{1}, y_{2}, \ldots, y_{n-1}$ of generators of the maximal ideal $\mathfrak{n}_{0}$ of $B_{0}$. Therefore $J=\left(y_{1}^{p}, \ldots, y_{r-1}^{p}, y_{r}, \ldots, y_{n-1}\right)$ and 
$\mathfrak{n}=\left(y, y_{1}, \ldots, y_{n-1}\right)$. Let $x_{i}$ be a lift of $y_{i}$ in $A$ for $i=1,2, \ldots, n-1$. Then $I=$ $\left(x^{p}, x_{1}^{p}, \ldots, x_{r-1}^{p}, x_{r}, \ldots, x_{n-1}\right)$ and $\mathrm{m}=\left(x, x_{1}, \ldots, x_{n-1}\right)$.

Acknowledgement. I express my deep gratitude to Prof. Balwant Singh, for his constant help and guidance.

\section{REFERENCES}

[1] L. Harper, On differentially simple algebras, Trans. Amer. Math. Soc., 100 (1961), $63-72$.

[2] T. Kimura and H. Niitsuma, On Kunz's conjecture, J. Math. Soc. Japan, 34 (1982), $371-378$.

[3] A. K. Maloo, Maximally differential ideals in positive characteristic, Comm. in Algebra, 20(8) (1992), 2365-2370.

[4] H. Matsumura, Commutative Ring Theory (Cambridge University Press, 1986).

[5] S. Yuan, Differentially simple rings of prime characteristic, Duke Math. J., 31 (1964), 623-630.

School of Mathematics

Tata Institute of Fundamental Research

Homi Bhabha Road, Bombay-400 005

INDIA 